\title{
Mexican species of Colpotrochia Holmgren, 1856 and Cubus Townes \& Townes, 1959 (Hymenoptera: Ichneumonidae: Metopiinae)
}

\author{
Мексиканские виды Colpotrochia Holmgren, 1856 и Cubus Townes \\ \& Townes, 1959 (Hymenoptera: Ichneumonidae: Metopiinae)
}

\author{
A.I. Khalaim ${ }^{1,2} \&$ E. Ruíz-Cancino ${ }^{1}$ \\ А.И. Хацаим, Э. Руис-Канцино
}

\begin{abstract}
${ }^{1}$ División de Estudios de Postgrado e Investigación, UAM Agronomía y Ciencias, Universidad Autónoma de Tamaulipas, Cd. Victoria 87149, México.

${ }^{2}$ Zoological Institute, Russian Academy of Sciences, Universitetskaya nab. 1, St. Petersburg 199034, Russia; E-mail: ptera@mail.ru

2 Зоологический институт РАН, Университетская наб., д.1, Санкт-Петербург 199034, Россия.
\end{abstract}

KEY WORDS: Ichneumonidae, Metopiinae, Colpotrochia, Cubus, Mexico, key.

КЛЮЧЕВЫЕ СЛОВА: Ichneumonidae, Metopiinae, Colpotrochia, Cubus, Мексика, определительный ключ.

ABSTRACT. Seven species of Colpotrochia and one species of Cubus occur in Mexico. Five of them, Colpotrochia catania, C. diabella, C. feroza, C. texana and $C$. watanka, are recorded for the first time from this country. The northern points of distribution of Colpotrochia concinna and Cubus validus are reported. Key to seven Mexican species of Colpotrochia is provided.

РЕЗЮМЕ. Семь видов Colpotrochia и один вид Cubus обитают в Мексике. Пять из них, Colpotrochia catania, C. diabella, C. feroza, C. texana и C. watanka, впервые указаны для этой страны. Приведены северные точки распространения Colpotrochia concinna и Cubus validus. Предложен определительный ключ для семи мексиканских видов Colpotrochia.

\section{Introduction}

Colpotrochia Holmgren, 1856 is a medium sized genus of Metopiinae with more than 60 described species distributed in all zoogeographical regions, except the Afrotropics [Yu et al., 2005]. Eighteen species of Colpotrochia occur in Costa Rica (15 of them were described from this country) [Gauld et al., 2002], four species occur in U.S.A. and Canada [Townes \& Townes, 1959], one species was described from Puerto Rico, and one species from Cuba. Only two species, C. concinna and $C$. mexicana, were recorded from Mexico till now [Townes \& Townes, 1966; Ruíz et al., 2002], both species were reported from southern Mexico. One more species, the widespread C. texana, is known to occur from southern Brazil to south of U.S.A., but it was not recorded from Mexico hitherto. The only species of the genus Cubus Townes \& Townes, 1959, C. validus, was known to occur from southern Brazil to southern Mexico [Gauld et al., 2002].
This work is based on the Ichneumonidae collection of the Universidad Autónoma de Tamaulipas in Cd. Victoria (Tamaulipas, México). Some additional specimens were obtained from the Instituto de Biologia, Universidad Nacional Autónoma de México (D.F., México). From this material, six species of Colpotrochia and Cubus validus have been recognized. Five of them, Colpotrochia catania, C. diabella, C. feroza, $C$. texana and $C$. watanka, are recorded from Mexico for the first time. Two species, Colpotrochia concinna and Cubus validus, have been found in Tamaulipas (Mexico); this is the northern point of distribution of these species. One more species, Colpotrochia mexica$n a$, occuring in Costa Rica and southern Mexico, is not found in our material. Key to seven known Mexican species of Colpotrochia is provided.

Key to Mexican sPeCies of ColPotrochia

1. Face yellow .............................................................. 2

— Face black ......................................................................... 3

2. Mesopleuron with a sharp and strongly raised subtegular ridge immediately below tegula (Fig. 6). First sternite posteriorly smooth. Labrum long and apically rounded, $0.4-0.5$ times as long as basally broad (Fig. 3) .... C. feroza Gauld et Sithole

- Mesopleuron below tegula flat, without a sharp raised subtegular ridge (Fig. 5). First sternite posteriorly corrugated, with distinct transverse rugae. Labrum short, apically truncated, $0.2-0.3$ times as long as basally broad (Fig. 2) ............................ C. diabella Gauld et Sithole

3. Fore wing with areolet present .................................... 4

- Fore wing without areolet ............................................ 5

4. Face, in anterior view, with labrum large, about half as long as basally broad (Fig. 1). Female with subgenital plate apically pointed (Fig. 7). Fore wing weakly and evenly infumate ................. C. catania Gauld et Sithole

- Face, in anterior view, with labrum small, at most 0.3 times as long as basally broad (Fig. 4). Female with subgenital plate apically truncated, scoop-shaped (Fig. 8 ). Mesoscutum more or less entirely black, at most only 

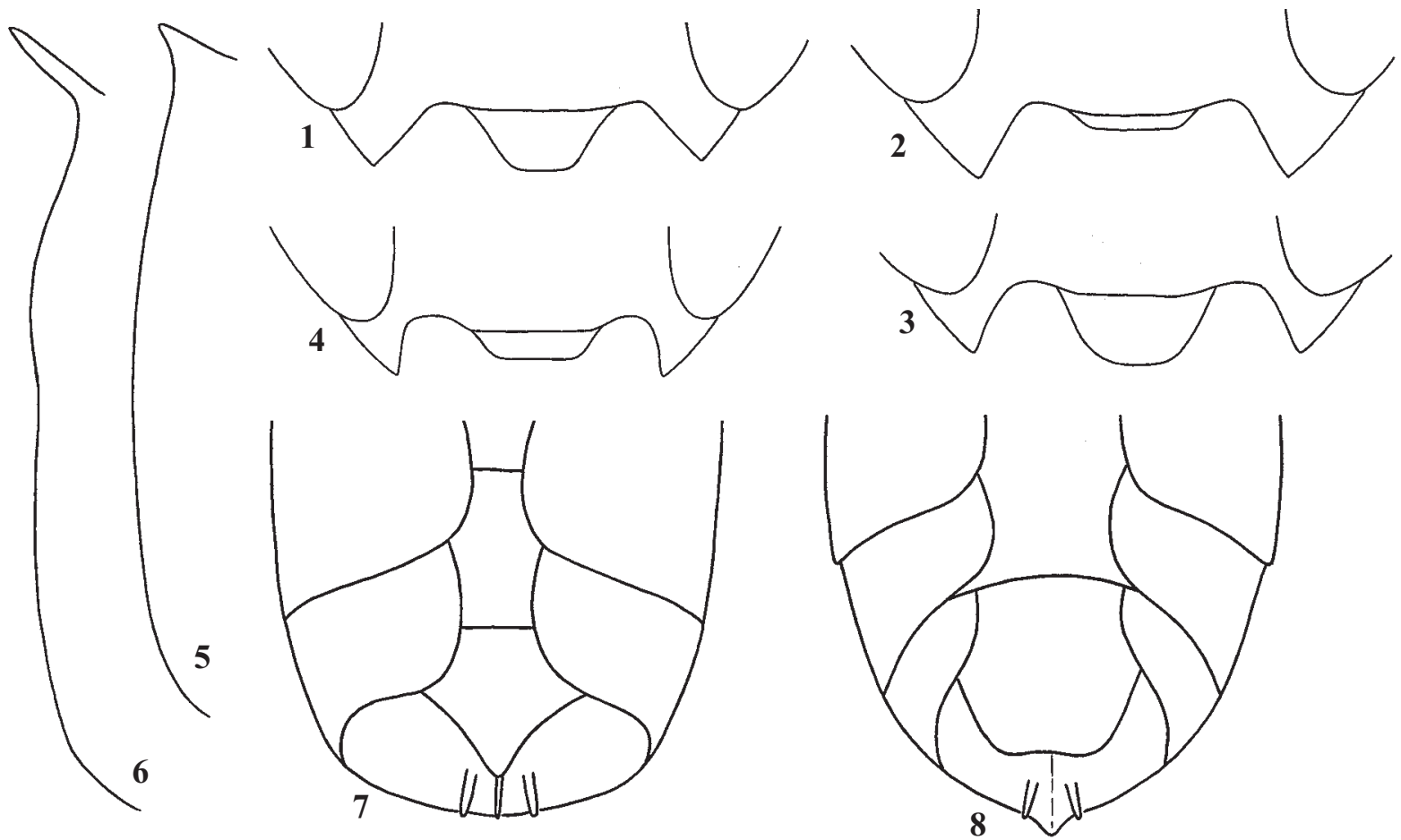

Figs 1-8. Colpotrochia spp., females: 1, 7 - C. catania; 2, 5-C. diabella; 3, 6-C. feroza; 4, 8-C. texana; 1-4- lower part of head with labrum, frontal view; 5-6 - contour of mesopleuron, frontal view; 7-8 - apex of metasoma with subgenital plate, ventral view.

Рис. 1-8. Colpotrochia spp., самки: $1,7-$ C. catania; 2, 5-C. diabella; 3, $6-$ C. feroza; 4, 8-C. texana; 1-4- нижняя часть головы с лабрумом, спереди; 5-6 - контур мезоплевры, спереди; 7-8 - вершина метасомы с субгенитальной пластиной, снизу.

narrowly yellow-marked along posterolateral margin ... C. texana (Cresson)

5. Mandible with lower tooth much longer and broader than upper tooth. Metasomal segment 2 with laterotergites broad, 1.4-1.6 times as long as wide, overlapping medioventrally along mid line..... C. mexicana (Cresson)

- Mandible with lower tooth subequal to upper tooth. Metasomal segment 2 with laterotergites moderately wide, 1.8-2.5 times as long as wide, not overlapping medioventrally ......

6. Propodeum black, posteriorly yellow-marked. Metasoma predominantly black, posterior 0.25 of tergite 2 and extreme posterior margins of following tergites yellow. Legs predominantly dark brown and black with few yellowish and brownish markings. Malar space $0.8-1.0$ times as long as basal width of mandible

\section{C. concinna (Cresson)}

- Propodeum yellow with extreme anterior margin fuscous. Metasoma with tergite 1 creamish brown in basal 0.7 , apically yellow, and tergites 2 and 3 fuscous in basal half and yellow in apical half. Legs yellow and creamish brown, internal surface of hind femur sometimes fuscous. Malar space in Mexican specimens about 0.5 times as long as basal width of mandible .. C. watanka Gauld et Sithole

\section{Colpotrochia catania Gauld et Sithole, 2002}

Figs 1, 7

MATERIAL. Mexico, Tamaulipas: $10^{7}, 40 \mathrm{~km}$ NW Cd. Victoria, Güémez, Cañon Salto del Tigre, 27.IV.2000, D.R. Kasparyan; $1 \sigma^{7}$, Cd. Victoria, Cañon del Novillo, 28.I.1985, A. del Valle; $2 O^{\top} O^{7}$, Cd. Victoria, Cañon Libertad [park Los Troncones], 6.VI.1985, B. Navarro D.; 1 +, Llera, 44 km S Cd. Victoria, matorral, Malaise trap, 28.X-4.XI.2000, D.R. Kasparyan; $10^{7}$, Gómez Farías, Alta Cimas, Malaise trap, 27.III-3.IV.1999, S. Hernán- dez A.; 2 우, same data, but 26.VI-10.VII.1999; 4 ○ึ $\bigcirc^{\top}$, Gómez Farías, Los Cedros, Malaise trap, 20.III-17.IV.1999, S. Hernández A.; 1 +, $2 \bigcirc^{7} O^{7}$, same data, but 22.V-19.VI.1999.

DISTRIBUTION. Costa Rica, Mexico (Tamaulipas). First record from Mexico.

\section{Colpotrochia concinna (Cresson, 1868)}

MATERIAL. Mexico, Tamaulipas: 1 , Gómez Farías, Alta Cimas, Malaise trap, 2.V-5.VI.1999, S. Hernández A.; $10^{\text {T, }}$, same data, but 31.VII-7.VIII.1999; 2 우, Gómez Farías, Los Cedros, Malaise trap, 26.XII.1998-7.I.1999, S. Hernández A.; 1 9, same data, but 13-20.II.1999; 1 \%, same locality, 27.II.1999, D.R. Kasparyan.

DISTRIBUTION. Costa Rica, Mexico (Córdoba in Veracruz, Tamaulipas).

Colpotrochia diabella Gauld et Sithole, 2002

Figs 2, 5

MATERIAL. Mexico, Nuevo León: $1+$, Monterrey, Malaise trap, 12.X.1983, M.R. Cantú; 1 †, S Monterrey, Parque La Estanzuela, 22.VIII.1986, E. Ruíz C. Tamaulipas: $10^{7}, 15 \mathrm{~km}$ NNW Cd. Victoria, Rancho Nuevo, 16.X.1998, D.R. Kasparyan; $10^{7}$, NW Cd. Victoria, Parque Los Troncones, 28.IX.1986, A. del Valle; $1 \mathrm{O}^{7}$, Cd. Victoria, La Libertad, 23.XI.1985, J. Hernández R.; 1 ○', Cd. Victoria, Cañon del Novillo, 800 m, 17.IV.1988, I. Villareal; 2 우, 2 O $^{\top} \mathrm{O}^{\top}$, same locality, 25.V-19.VII.1985, Serna, A. del Valle; $1 O^{7}$, Cd. Victoria, 25.VI.2008, E. Ruíz C.; 1 +, $1 \sigma^{7}, 15 \mathrm{~km}$ SSE Cd. Victoria, El Madroño, 8.VII.1984; $1 \mathrm{O}^{7}$, same data, but 10.VII.1985; $1 \mathrm{O}^{7}, 25 \mathrm{~km}$ SSW Cd. Victoria, Altas Cumbres, 31.V.1998, D.R. Kasparyan; 1 +, Jaumave, 22.IX.1989, M. Aquino S.; 1 \%, Ocampo, 27.IV.1989, E. Ruíz C.; 1 †, 1 O', Gómez Farías, Canindo, Malaise trap, 20-22.VII.1994, J.B. Woolley.

DISTRIBUTION. Costa Rica, Mexico (Nuevo León, Tamaulipas). First record from Mexico.

BIOLOGY. Reared from Melipotis fasciolaris (Hübner, 1823) (Noctuidae) on Quercus oleoides in Costa Rica [Gauld et al., 2002]. 
Colpotrochia feroza Gauld et Sithole, 2002

Figs 3, 6

MATERIAL. Mexico, Tamaulipas: 2 우, Gómez Farías, Alta Cimas, 2.XI.1990, E. Ruíz C.; 1 + , same locality, Malaise trap, 26.VI-3.VII.1999, S. Hernández A.; $1 \sigma^{7}$, same data, but 2128.VIII.1999; $1 \mathrm{O}^{7}$, same locality and trap, $900 \mathrm{~m}, 4-12$.VIII.2000, D.R. Kasparyan; 1 ㅇ, Gómez Farías, Los Cedros, Malaise trap, 27.II-6.III.1999, S. Hernández A.; $1 \sigma^{7}$, same data, but $10-$ 17.IV.1999. San Luis Potosí: 1 + , Xilitla, Las Pozas, 21-23.XII.2008, A.I. Khalaim.

DISTRIBUTION. Costa Rica, Mexico (San Luis Potosí, Tamaulipas). First record from Mexico.

Colpotrochia mexicana (Cresson, 1868)

DISTRIBUTION. Costa Rica, southern Mexico (Córdoba in Veracruz).

\section{Colpotrochia texana (Cresson, 1872) \\ Figs 4,8}

MATERIAL. Mexico, Tamaulipas: 1 , NW Cd. Victoria, Parque Los Troncones, 26-31.XII.2008, A.I. Khalaim; 1 + , Gómez Farías, Alta Cimas, Malaise trap, 19-26.VI.1999, S. Hernández A.; 1 ㅇ, $1 \mathrm{O}^{\top}$, Gómez Farías, Los Cedros, Malaise trap, 17.IV-8.V.1999, S. Hernández A.

DISTRIBUTION. Southern Brazil, Costa Rica, Mexico (Tamaulipas), south east of U.S.A. (North Carolina to Florida and Texas). First record from Mexico.

\section{Colpotrochia watanka Gauld et Sithole, 2002}

MATERIAL. Mexico, Oaxaca: $1 \uparrow, 1 \sigma^{7}$, Estación Biologica Los Tuxtlas, 61 m, 19.41925 N, 104.97326 W, Malaise trap, 112.XI.2009, A. Zaldivar.

VARIABILITY. According to the original description [Gauld et al., 2002], two Mexican specimens differ from material from Costa Rica by the shorter malar space $(0.5$ times as long as basal width of mandible in Mexican specimens and 0.6-1.0 times in Costa Rican material), and metasomal tergites 4-6 yellow and black banded (tergites 4+ in specimens from Costa Rica brownish black).

DISTRIBUTION. Costa Rica, Mexico (Oaxaca). First record from Mexico.
Cubus validus (Cresson, 1865)

MATERIAL. Mexico, Tamaulipas: $10^{7}$, SE Cd. Victoria, Rancho Sta. Elena, $23^{\circ} 37.362^{\prime} \mathrm{N}, 99^{\circ} 12.746^{\prime} \mathrm{W}, 970 \mathrm{~m}, 8-14 . V .2010$, E. Ruíz C., J.M. Coronado B.; 1 + Cd. Victoria, Cañon del Novillo, 22.IX.1984, E. Ruíz C.; 3 우, 1 ○', same locality, 8.VI-15.VII.1985, A. del Valle; $10^{7}$, same data, but 2.VIII.1985. Veracruz: $10^{7}$, San Andrés Tuxtla, 20.xii.1984, H. Brailovsky.

DISTRIBUTION. Southern Brazil, Colombia, Costa Rica, Cuba, Mexico ("southern Mexico", Veracruz, Tamaulipas).

BIOLOGY. Reared from Eulepte concordalis (Hübner, 1826) (Pyralidae) on Xylophragma seemannianum (Bignoniaceae), Omiodes humeralis Guenée, 1854 (Pyralidae) on Inga vera (Fabaceae), and Pleuroptya silicalis (Guenée, 1854) (Pyralidae) on Cecropia peltata (Cecropiaceae) in Costa Rica [Gauld et al., 2002].

ACKNOWLEDGEMENTS. We are thankful to Dr. A. Zaldivar (Instituto de Biologia, Universidad Nacional Autónoma de México, D.F., México) for loan of valuable material. This work was supported by the PROMEP Project "Taxonomia y ecologia de fauna y micobiota en comunidades forestales y cultivos", the Russian Foundation for Basic Research (no. 10-04-00265) and the Presidium of the Russian Academy of Sciences Program "Origin and evolution of Biosphere, Subprogram II".

\section{References}

Gauld I.D., Sithole R., Gómez J.U. \& Godoy C. 2002. The Ichneumonidae of Costa Rica, 4 // Memoirs of the American Entomological Institute. Vol.66. P.1-768.

Ruíz E.C., Kasparyan D.R. \& Coronado Blanco J.M. 2002. 37. Ichneumonidae // J. Llorente Bousquets, J.J. Morrone (eds.). Biodiversidad, Taxonomía y Biogeografía de Artrópodos de México: Hacia una síntesis de su conocimiento. México: Conabio-Ecosur-Bayer. P.631-646.

Townes H.K. \& Townes M. 1959. Ichneumon-flies of America north of Mexico: 1. Subfamily Metopiinae // United States National Museum Bulletin. Vol.216. P.1-318.

Yu D.S., van Achterberg K. \& Horstmann K. 2005. World Ichneumonoidea 2004. Taxonomy, Biology, Morphology and Distribution. CD/DVD. Taxapad, Vancouver, Canada. www.taxapad.com 